\title{
Complexity in nuclear materials
}

\author{
In the nuclear industry, safety considerations rely on our ability to understand and control the behaviour \\ of the relevant materials over a range of length and time scales.
}

Nuclear power remains a contentious issue for many. Its proponents point towards it being a cost-competitive, feasible route for meeting our ever growing appetite for energy, with relatively low greenhouse gas emissions (Nature Mater. 7, 679; 2008). Those against a nuclear-led energy policy cite concerns over the devastating impact a nuclear incident can have, as well as the complex scientific and societal issues that are faced in deciding what to do with radioactive waste. At the heart of many of these issues are challenges based on complex materials science - in particular, the behaviour of materials over a wide range of spatial scales and in the timeframe of hundreds of thousands of years - that require careful consideration. Four years since the accident at the Fukushima Daiichi nuclear plant in Japan, in this issue of Nature Materials we take a careful look at some of the materials-related challenges that are critical to the nuclear power industry.

In the event of a nuclear incident, such as those at the Fukushima Daiichi, Chernobyl and Three Mile Island nuclear plants, it is vital that the amount and chemical nature of the released radioactive material is identified, so that the likely impact on the surrounding environment can be predicted. In a Commentary on page 247, Rudy Konings and colleagues discuss the mechanistic processes that control the release of fissile material from a damaged reactor. They highlight the length scales and processes that must be accounted for, in particular, those related to the atomic-scale diffusion of fissile material through the $\mathrm{UO}_{2}$ fuel lattice, the micromechanical integrity of the zirconiumalloy cladding that contains the fuel, and the interactions of the released radionuclides with the surrounding water coolant and reactor-containment barriers.

Devising suitable approaches for the longterm geological storage of spent nuclear fuel likewise involves multiple material systems and processes, which are no less complicated. As Rodney Ewing discusses in a second Commentary on page 252, critical to the storage of spent $\mathrm{UO}_{2}$ fuel is knowledge of the evolution of radiological characteristics and, more importantly, the effect that this has on the individual processes that influence the surrounding material systems. Indeed, the chemistry and radiotoxicity of the spent fuel evolves; highly radioactive fission products

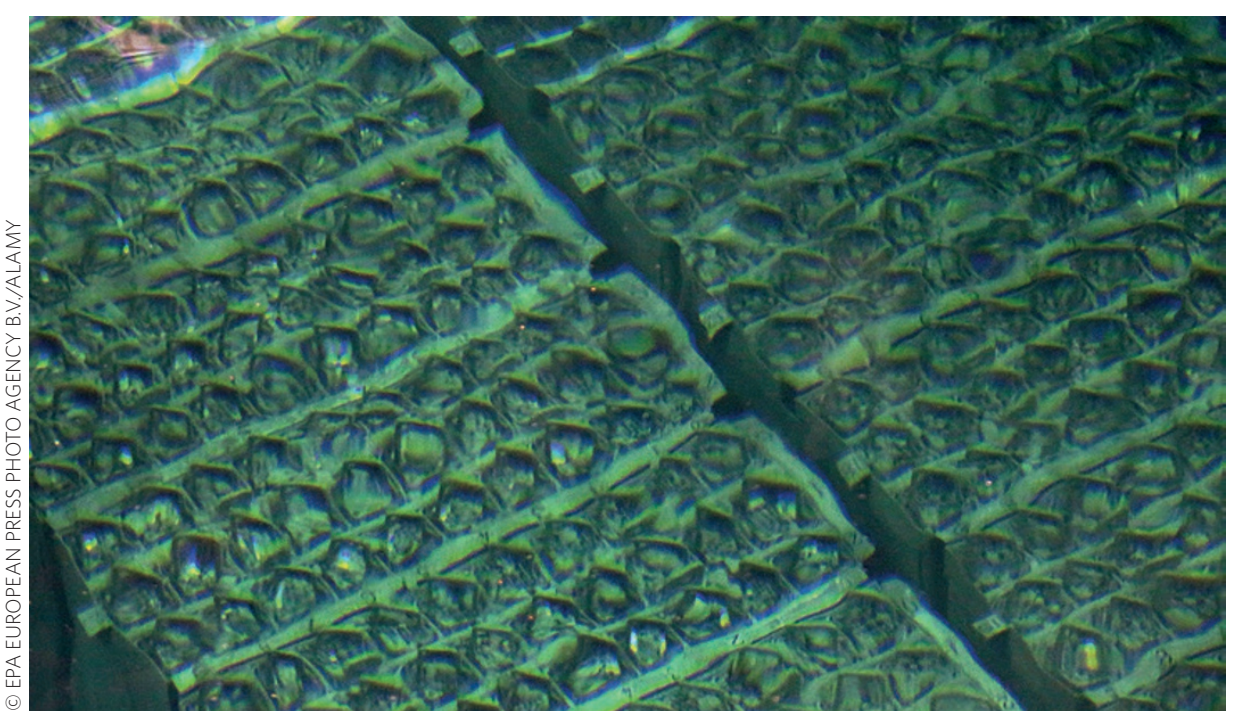

Fuel rods from reactor Unit 4 at the Fukushima Daiichi nuclear power plant in a spent-fuel water pool.

initially dominate, with half-lives measured in decades, but with time this shifts towards actinides, whose half-lives can stretch into millions of years. Ensuring the integrity of geological storage sites therefore requires that the time-dependent nature of the fuel and, crucially, how the changing chemistry affects interactions with the multi-barrier layers in storage repositories, be understood.

High-level waste from reprocessing of nuclear fuel is commonly immobilized in a borosilicate glass, to isolate hazardous fission products and minor actinides from the environment. The chemical durability and mechanical integrity of borosilicate glasses make them an attractive option for this, where the active radionuclides become part of the glass-network structure. However, it is well known that these wasteforms are not immune to chemical attack, for instance, during exposure to stagnant groundwater in a geological storage site. Under such conditions, a chemically altered layer forms at the surface region of a vitreous wasteform, which has long been associated with a diffusion-based mechanism. However, on page 307 Roland Hellmann and co-authors report, on the basis of atomic-scale structural and chemical analyses, that the interface between the chemically altered region and the pristine bulk in a borosilicate glass is sharp on the nanometre scale, which is at odds with the long-assumed diffusion-dominated process. Andrew Putnis discusses this point in an accompanying News and Views on page 261.

It is apparent then that there are a host of materials-related challenges that play key roles in achieving a safe and efficient nuclear industry. In few places are these issues more in the public eye than in Japan, where the first nuclear reactors since the accident at Fukushima Daiichi may soon be brought back online. In an Interview on page 258, Tatsuo Shikama, Director of the International Research Centre for Nuclear Materials at Tohoku University, talks about the present state of play of nuclear materials in Japan, and the challenges that the country, and the nuclear industry as a whole, face.

The discussions presented in this focus issue point towards it not being enough to simply understand the individual scientific mechanisms at play when trying to design and control the complex multi-material systems utilized in nuclear reactor cores and in proposed geological disposal sites. Instead, it is vital that the relationships between the various operative mechanisms and materials are understood over a range of length scales and as a function of time, so that accurate predictive models can be built. Such predictive capabilities are important for ensuring a safe nuclear industry and future environmental protection. 